\title{
A compiled catalogue of reference stars around the selected ERS in the northern sky
}

\author{
V. P. Ryl'kov ${ }^{1}$, N. Narizhnaja ${ }^{1}$, A. Dement'eva ${ }^{1}$, N. Maigurova ${ }^{2}$, G. I. \\ Pinigin $^{2}$ and Y. Protsyuk ${ }^{2}$ \\ ${ }^{1}$ Main Astronomical Observatory of RuAS, MAO RuAS, Russia \\ email: vryl@gao.spb.ru; \\ ${ }^{2}$ Nikolaev Astronomical Observatory, NAO, Ukraine \\ email: pinigin@mao.nikolaev.ua
}

\begin{abstract}
A compiled catalogue consisting of more than 22000 reference stars in the range of $10^{m}-16^{m}$ was obtained with 235 fields of $\delta$ from $-17^{\circ}$ to $+80^{\circ}$ around extragalactic radio sources (ERS) selected from the ICRF list. Initial catalogues were obtained from photographic $(\mathrm{PG})$ and CCD observations.
\end{abstract}

Keywords. astrometry, reference systems

\section{Observational data}

One of the important problems of ground-based positional astrometry is the refinement of link between the radio and optical coordinate systems (de Vegt et al. 2001, Ryl'kov et al. 2005, Ryl'kov et al. 2005). A compiled catalogue consisting more than 22000 reference stars (RS) in the range of $10^{m}-16^{m}$ with 235 fields of $\delta$ from $-17^{\circ}$ to $+80^{\circ}$, centered in the optical CCD-image of faint astrometric ERS (ICRF ERS) was obtained for a link to VLBI-observations (ICRS system). For this purpose 4 original star catalogues were used. Three of them were obtained by PG method at the astrograph (AG) telescopes, the rest at the Axial meridian circle (AMC) with a CCD camera.

Pul ERS. PG observations were obtained at the Normal AG (330/3464) in Pulkovo observatory in the 1990s. There are 74 ERS fields (35 are processed by now) in $\sim 300$ plates. The number of stars in the catalogue is $\sim 4500$. They were obtained in a field of $20^{\prime}$ radius around ERS. The limiting magnitude is $17^{m}$.

PIRS-K. Kiev (PG Intermediate Reference Stars) Catalogue. The observations were carried out for 115 ERS fields (2875 stars) with the 200/4126mm AG of Kiev University. In one field there are $25 \mathrm{RS}$ in average within the field of $1^{\circ}$ diameter centered in ERS.

PIRS-B. Bucharest. The observations were made at the $380 / 6000 \mathrm{~mm}$ double AG in Romanian National Observatory in Bucharest using the field of $60^{\prime}$ and 188 ERS fields ( 4700 stars $)$ were photographed in the 1990s.

AMC1B. The CCD observations were made in the near of ERS at the AMC 180/2480 $\mathrm{mm}$ of NAO. The field $(\alpha \times \delta)$ is $60^{\prime} \times 24^{\prime} .208$ ERS fields $(\sim 17000$ stars $)$ were obtained. The AMC catalogue was compiled using the UCAC2 catalogue as reference one.

It was decided to sum up these catalogues, made approximately in the same epoch, for increasing density and accuracy of positions of stars around ERS. It was necessary to bring their positions to the same epoch and to study differences of coordinates of the stars for decreasing the random and systematic deviations because these catalogues were obtained with different telescopes and even by different methods of image registration.

The stars, without proper motions in initial catalogues (IC), were processed in the following way. We believe that the positions from the IC may be averaged, because the 
faint stars have small proper motions and the difference of epoch of observations does not exceed 10 years. Proper motions of stars were taken from the UCAC2 (N.Zacharies et al. 2000) to study the systematic deviations of the significant part of catalogue stars.

\section{Forming and research of compiled catalogue}

Comparison of positions for common stars in these IC was made. The stars with large differences in positions were excluded from the IC. About 30000 of star measurements were processed in total from four catalogues. More than 10000 stars in 151 fields around ERS with proper motions of the UCAC2 were found by averaging; 11000 stars with no proper motion data were averaged and included in the compiled catalogue. All stars which have proper motions (in $\delta$ up to $+45^{\circ}$ ) were reduced to the epoch and equinox J2000, for the rest positions have the mean epoch of observations and equinox J2000.

Thus, we obtained the catalogue of positions for 21355 basic stars in 235 fields of $20^{\prime}-30^{\prime}$ radius with ERS. Almost 10650 stars have proper motions from the UCAC2. At present the compiled catalogue is not homogenous because many fields contain few RS, less than ten. They are mainly fields which were not included into programs of observation in Pulkovo and Nikolaev. They will be extended with observations in future.

The individual catalogues of each field compiled by us should be checked up for errors by comparison, at least, with 2 independently compiled catalogues. We chose these:

- the UCAC2, which has stars brighter than $16^{m}$ in $\delta$ up to $+40^{\circ}-+50^{\circ}$;

- the CMC13 (Carlsberg Meridian Circle, N13) for stars in $\delta$ from -3.2 to +30.2 .

At first we chose ten fields in declinations from $0^{\circ}$ to $+30^{\circ}$, where the number of common stars is great for studying the accuracy of the compiled catalogue. It is evident that the systematic deviations in $\alpha$ and $\delta$ exist. It should be noted, that they are less than 100 mas in $\alpha$, but reach 200 mas in $\delta$ in several fields. If you take into account that the comparison catalogues (CMC13 and UCAC2) have both internal and external errors of $50-100$ mas for $12^{m}-16^{m}$ stars, then the compiled catalogue is in good agreement with the accuracy limits. The majority of the stars have the photometry of $12^{m}-15^{m}$ from the UCAC2 magnitude and accuracy scatter in the limits \pm 0 ". 5 for both coordinates.

If you take into account that stars without identification with the UCAC2 are mainly faint, it is possible to consider that the centre value of distribution from magnitude in range of $14^{m}-15^{m}$ is based to the fainter stars. We found mean values of deviations for the coordinates of RS from their positions in the UCAC2. There were reduced all common stars for 200 fields with ERS. Several fields were compared with CMC13.

One can make a conclusion from the comparison, that several fields have significant deviations in $\alpha$ and $\delta$. These circumstances indicate requirements of regular observations and improvement of stellar positions of the reference catalogues for obtaining the positions of ERS.

The research results of the compiled catalogue for 235 fields with ERS is available on magnetic disks in laboratory of astrometry of MAO RuAS and NAO, Ukraine.

\section{References}

de Vegt, C., Hindsley, R., Zacharias, N., \& Winter, L. 2001, A.J., 121, 2815-2818

Ryl'kov, V., Dement'eva, A., Narizhnaya, N., Pinigin, G., Maigurova, N., Protsyuk, Yu., Bocsa, G., Popescu, P., \& Kleschenok, V. 2005, Kinematics and Physics of Celestial Bodies, Suppl., $5,328-332$

Ryl'kov, V., Dement'eva, A., Narizhnaya, N., Maigurova, N., Pinigin, G., Protsyuk, Yu., Bosca, G., \& Popescu, P., 2005, Romanian Astronomical Journal, Suppl., 15, 131-136 